\title{
Factors influencing participation rates in clinical late effect studies of childhood cancer survivors
}

\author{
Anne Sophie Helligsoe ${ }^{1}$, Louise Henriksen ${ }^{1}$, Line Kenborg ${ }^{2}$, Christian Dehlendorff ${ }^{2}$, \\ Jeanette Winther ${ }^{2}$, and Henrik Hasle ${ }^{1}$ \\ ${ }^{1}$ Aarhus University Hospital \\ ${ }^{2}$ Danish Cancer Society Research Center
}

January 20, 2021

\begin{abstract}
Introduction: Several studies on late effects of childhood cancer have been conducted during the past decades. To ensure external validation of a study population, the participation rate must be high. This study investigated demographic data in late effect studies and potential factors impacting on participation rates such as cancer type, time since diagnosis and duration of clinical examinations. Procedure: By searching the databases PubMed, Embase and Web of Science and by contacting researchers and clinicians, we identified studies including an invitation to a clinical examination for late effects after childhood cancer. Studies conducted from January 2010 - March 2020 in the Nordic countries were included. Results: We found 80 published studies originating from 16 cohorts. The overall participation rates ranged between 27 and 100\%. The majority of studies (eleven studies) were conducted more than ten years after the cancer diagnosis and primarily on hematologic malignancies (seven studies). The highest participation rates were seen in studies of survivors with solid tumors (92\%) and the lowest in survivors with hematologic malignancies $(67 \%)$ and central nervous system tumors $(73 \%)$. Neither duration of the clinical examination nor time since diagnosis seemed to affect the participation rate. Conclusion: A trend of lower participation rates when recruiting survivors of hematologic malignancies and central nervous system tumors was found. We encourage future studies to describe the recruitment process more thouroughly to improve understanding of the factors influencing participation rates.
\end{abstract}

\section{Introduction}

In the Nordic countries, approximately 660 children below 15 years are diagnosed with cancer annually [1]. During the past decades, improvements in surgical interventions, radiation and/or chemotherapy have contributed to a major improvement in the five-year survival now exceeding $80 \%$ for all childhood cancers combined in most high-income countries [1]. Due to improved survival, the population of childhood cancer survivors is steadily growing and it has been estimated that approximately one in 1000 adults in the general population is now a childhood cancer survivor [2].

Cancer survivors face a life with an increased risk of a wide range of somatic and mental late effects related to their cancer diagnosis and therapeutic exposures with a cumulative burden nearly two-fold greater than controls $[3,4]$. Several clinical studies of late effects of childhood cancer have been conducted during the past decade. To obtain relevant, comparable and generalisable results from the clinical studies, the population must be representative of the survivors. Studies with low participation might lack adequate statistical precision and thus might be inconclusive or subject to selection bias. Factors known to influence recruitment procedures and response rates have repeatedly been of interest $[5,6]$. Potential barriers for participation in clinical late effect studies were analyzed in the Childhood Cancer Survivor Study (CCSS) [4], which identified distance to the hospital and fear of bringing back old memories of being sick as the most important barriers. Among motivating factors were re-visiting clinicians who had been involved in their treatment, to learn more about their own health, and helping out other children with cancer. 
Recruitment of especially adolescents and young adults (AYAs), however, can be challenging. A study investigating recruitment of AYAs between 15-39 years in the United States through a survey achieved a response rate of $43 \%$ [7]. Although this survey invitation was sent close to the date of diagnosis, it required extensive resources to achieve a sufficiently high participation rate in this age group of young people being mobile and difficult to follow due to educational and employment opportunities, marriage and other personal life changes.

A systematic review assessed participation reporting quality, participation percentage and characteristics in psychosocial oncology studies including both adult and pediatric patients [8]. Of the 156 pediatric studies included, $121(78 \%)$ reported sufficient data to calculate a participation rate, which was higher in studies conducted near the time of diagnosis compared to later in the treatment trajectory.

Willingness to participate in a clinical study seems to vary markedly among childhood cancer survivors [9] [10], which might be due to a variety of different factors influencing participation. However, this has not previously been systematically investigated in childhood cancer survivors. Thus, our aims were to outline the participation rate among childhood cancer survivors and to investigate potential factors influencing the participation rate, including cancer type of the survivor, time since diagnosis, and duration of the clinical examination. We investigated wether survivors with a cancer diagnosis carrying a high risk of late effects such as hematologic malignancies and central nervous system tumors, long time since diagnosis, and a long duration of the clinical examination would lead to a lower participation rate.

\section{Methods}

\section{Literature search}

A comprehensive search strategy was developed from systematic reviews in collaboration with a health science librarian. We combined MeSH headings for cancer, children, survivor/long-term and Nordic countries supplemented with free text words. Further details on search strategies are provided in Supplemental Material. Relevant studies were identified by searching the databases PubMed, EMBASE and Web of Science and by manual review of reference lists in full-text articles as well as the clinical trials register, clinicaltrials.gov. Studies were screened based on title and abstract according to inclusion and exclusion criteria as described below.

\section{Study selection criteria}

We searched for studies in English published from January $1^{\text {st }} 2010$ - March $1^{\text {st }} 2020$ conducted within the Nordic countries (Denmark, Norway, Sweden, Finland and Iceland) and including an invitation to a clinical examination for assessment of late effects after childhood cancer. Participants had to be younger than 18 years at time of the cancer diagnosis, whereas no requirements to the minimum size of the study population was set up. The clinical examination should have been performed at least three years after the cancer diagnosis and could include any type of clinical examination such as echocardiography, endocrinologic or neurocognitive testing or radiologic imaging. Studies were excluded if no clinical examination was performed in the study population, and if data were solely based on questionnaires, blood samples, telephone interviews, online focus group interviews, or routine out-patient follow-up visits. We also excluded studies if part of the study population was above 18 years of age at diagnosis, or if the study was conducted outside the Nordic countries. Furthermore, reviews, surveys, guidelines or editorial letters were excluded in addition to posters and conference abstracts, since they typically do not describe the recruitment process in deltail. If more studies on the same cohort were published, only the first study published on the cohort was included.

\section{Data extraction}

Eligible full text studies were included if they met the predefined inclusion criteria. For each study, the following data were abstracted for the study population: participation rate, cancer type, sex distribution, time since diagnosis or stem cell transplantation $(\mathrm{SCT}$ ), duration of the examination (estimated to more or less than three hours), the person who contacted the participant (doctor, nurse or other) and how the participant was approached (postal letter, mail or telephone). Four predefined diagnostic groups of childhood 
cancer were used: Hematological malignancies, central nervous system tumors, solid tumors, and mixed tumors defined as more than one diagnostic group. In case of missing data, corresponding or senior authors were contacted to obtain additional information.

Definitions of participation rate

Invited participants were defined as survivors receiving an invitation to participate in a late effect study including a clinical examination. Invited participants were further divided into accepted participants (invited participants who accepted the invitation), study non-participants (invited participants who declined or did not respond to the invitation), study dropouts (invited participants who accepted the invitation but did not complete the clinical examination) and study completers (invited participants who accepted the invitation and completed the clinical examination).

Statistical analysis

The study participation rate (SPR) was calculated as the ratio between the number of accepted and invited participants. In case-control studies, only the SPR for the cases was calculated and not for the controls. To address the potential effect of participant characteristics, we used a random-effect logistic regression model for the logit-transformed (log odds) participation rates. For studies with a $100 \%$ participation rate, the number of non-participants was set to 0.5. We investigated cancer type (hematological malignancies, central nervous system tumors, solid tumors or mixed diagnosis), time since diagnosis (more or less than ten years) and duration of the examination (more or less than three hours), separately. The results were presented in forest plots in which the logit-transformed participation rates were transformed back to the participation rate-scale with corresponding $95 \%$ confidence intervals (CI).

\section{Results}

We initially identified 2080 studies and 80 studies met the selection criteria. For flowchart of the study screening and inclusion, see Figure 1. The authors of these 80 studies had all published several studies originating from the same childhood cancer cohort. Including only the first study published on the cohort resulted in 16 studies each representing different childhood cancer cohorts (Table 1): one Nordic study (Denmark, Norway, Sweden, Iceland and Finland) [11]; three in Norway (two based in a single center [12, 13] and one national [14]); five in Sweden (all in a single center [15-19]); six in Finland (three in a single center [20-22] and three national cohorts [23-25]); and one in a single center in Iceland [26]. The full study sample based on the respective 16 cohorts identified included 879 childhood cancer survivors comprising 341 females and 538 males.

Recruitment procedures of the person who contacted the participant (physician, nurse or other) and how the participant was approached (postal letter, mail or telephone) were not consistently reported. In six cohort studies, a letter was send to the participants, whereas in six other cohort studies a physician was the direct contact person. Information on the female-male ratio among the invited participants, study drop-outs, and study non-participants was not described in the studies. Only sex distribution of the accepted participants was available. Two male-only studies investigated testicular function, whereas 14 studies comprised both males and females.

Clinical examinations included training programs, endocrinologic or audiologic evaluations, radiologic imaging and neurocognitive evaluations. The number of participating females was highest in patients with solid tumors (19 females of 34 participants, 55.8\%) and mixed diagnosis (42 females of 77 participants, 54.5\%) compared to those with hematologic malignancies (174 females of 358 participants, $48.6 \%$ ) and central nervous system tumors (106 females of 223 participants, 47.5\%). Further characteristics of the participants in the included studies are displayed in Table 1.

SPR ranged from 27 to $100 \%$ (median $72.5 \%$ ) (Figure 2). The lowest SPR was registered by Järvela et al. $(27 \%)$ [27], who investigated physical activity with a home-based training program for survivors of acute lymphoblastic leukemia. The highest SPR being $100 \%$ was reported by Einarsson et al. [19] and Einar-Jon et al. [26] both performing audiometric evaluation of survivors receiving platinum-based chemotherapy. 
The age at diagnosis ranged from 3.0 to 14 years (median 6.0). The median age at diagnosis was lowest for studies investigating late effects of acute myeloid leukemia (median 3.0 years, range 0-15) [11]. The highest median age at diagnosis was 14 years (range 0-18) and at study entry 32 years (range 19-55) observed in an interview study involving clinical examination of late effects after malignant lymphoma [28]. The lowest age at study entry was observed in a study of neurocognitive late effects after acute lymphoblastic leukemia (median 11.5 years, range 8.4-15.3) [12].

\section{Cancer type, time since diagnosis and duration of the clinical examination}

Three studies included mixed diagnosis; i.e., one study being an audiologic evaluation of cancer survivors who had received platinum-based chemotherapy [10], another conducting a sperm analysis [29], and the last performing a cardiac Magnetic Resonance Imaging on survivors who had received anthracyclines [22]. Five studies included patients with previous hematologic malignancies with an examination duration of more than three hours and a median follow-up time of more than ten years. Studies on solid tumors had shorter duration and either a long or missing follow-up time.

\section{Cancer type}

By grouping the studies into four predefined diagnostic groups (hematologic malignancies, central nervous system tumors, solid tumors and mixed), we analyzed SPR related to cancer diagnosis (Figure 3). We included 16 cohorts of survivors with hematological malignancies $(n=7)$, CNS tumors $(n=4)$, solid tumors $(n=2)$, and mixed diagnosis $(n=3)$. The studies of mixed diagnosis were quite heterogenous. No statistically significant difference in SPR in regard to diagnosis was found $(\mathrm{p}=0.33)$, although we found a tendency to a higher participation in late effect studies of solid tumors compared to hematologic malignancies.

\section{Time since diagnosis}

Time since diagnosis was more than ten years in eleven studies and less than ten years in five studies (Figure 4). No statistically significant difference was found with SPR in time since diagnosis $(p=0.99)$. There was considerable heterogeneity within each group of time since diagnosis.

\section{Duration of the clinical examination}

Duration of the clinical examination was separated into examinations with a duration of more or less than three hours, respectively, and SPR was analyzed accordingly (Figure 5). In ten studies, the clinical examination lasted more than three and in six studies less than three hours. No statistically significant difference in SPR was found for duration of examination ( $\mathrm{p}$-value $=0.42$ ).

\section{Discussion}

This analysis of participation rates in late effect studies after childhood cancer illustrates potential factors influencing participation rates. We included 879 survivors of childhood cancer characterized by universal access to tax-payed health care, low social inequality, access to population-based cohorts and complete follow-up using highly valid and complete registers by use of the civil registration number assigned to all citizens. We applied a systematic search strategy and sought additional information from the corresponding authors in case of missing data.

All studies reported SPR or provided information sufficient to calculate SPR. Three studies did not clarify study dropouts. Less than three studies had missing data on age distribution. Study populations ranged from 15-161 participants, and the outcome measures varied with diverse clinical examinations such as exercise programs, endocrinologic or audiologic evaluations, radiologic imaging and neurocognitive examinations. SPR ranged from $27-100 \%$. The two studies with complete participation were rather small studies each including 15 patients.

Importantly, SPR varied between diagnostic groups being lowest when inviting survivors of central nervous system tumors (73\%; 95\% CI [0.63-0.81]) or hematologic malignancies (67\%; 95\% CI [0.52-0.79]). A tendency towards a higher SPR in studies of solid tumors compared to hematological malignancies may be due to a 
different burden of late effects. Although survivors of solid tumors have an increased risk of late effects [30, 31], a higher risk is reported in survivors of hematologic malignancies [32, 33] or central nervous system tumors [34]. Although the extent of cognitive impairments can be different in the two patient groups, attention deficits and impairments in executive functioning among survivors of pediatric acute lymphoblastic leukemia and central nervous system tumors have been identified $[35,36]$. Executive functions are cognitive high-level skills, controlling, organizing and planning neurocognitive activity such as behavioral actions and social learning. Thus, pediatric cancer survivors may be challenged to read an invitation letter and take the initiative to reply, due to impaired planning and organizing skills; this could lead to a lower participation rate.

Attendance in late effect clinics has been widely investigated [37, 38]. Nathan et al. [39] found increased rates of attendance associated with female gender and higher socieoeconomic status. In our cohorts, information on sex distribution in the invited participants was not available, and therefore we could not estimate the sex distribution in invited compared to the sex distribution of participants accepting to be enrolled in the study. Further, as recruitment methods were inconsistently reported, we were not able to investigate if contact by telephone or letter influenced the participation rate. Harlan et al. [7] described that extensive efforts were necessary to recruite adolescents and young adults to complete a questionaire. In the future, we need to consider more personalized recruitment strategies, as study populations in late effect studies are heterogenous also regarding age.

We divided the examinations in the studies to be of either short (less than three hours) or long (more than three hours) duration to evaluate if a long examination was a potential barrier for participation. Studies on solid tumors had small samples sizes and always a short duration of clinical examination and either a long or missing follow-up time. This might result in discrete changes in small sample sizes being possibly of disproportionate importance. Five studies included hematologic cancers with a long duration and long median follow-up time of more than ten years. This implies that marginal analyses considering one factor at a time may be explained or confounded by other factors. The five studies of mixed tumors were quite heterogenous considering participants and duration of clinical examinations resulting in a more imprecise pooled estimate for this group.

We did not find any statistically significant differences in time since diagnosis and duration of examination in our study. The majority of participants were invited more than ten years after being diagnosed, and we found that neither time since diagnosis nor the duration of the examination were challenging in recruitment.

Underreporting of recruitment strategies also made it difficult for Hudson et al.[40] to evaluate the impact of different recruitment strategies. They encouraged transparency in the reporting of participant identification, invitation and consent to enable researchers to understand research implications, risk of bias and to whom results apply [40]. This is additional information compared with the current structured guidelines for observational studies [41].

Although it has been clear for more than four decades that lifelong survivorship care is needed for most survivors, there is a great variability in the provision of long-term follow-up programmes for childhood cancer survivors across countries and thus still an urgent need for improvement in long-term follow-up care of survivors [42, 43]. Even in the Nordic countries, where late effect clinics are more widely distributed than in many other countries, a low participation rate might lead to little or no follow-up of childhood cancer survivors and thus the risk of an increased morbidity in childhood cancer survivors.

We encourage future clinical late effect studies to describe the recruitment procedure more thoroughly including sex distribution of the invited patients, how patients were contacted and by whom. We believe that this will lead to a better understanding of the factors influencing participation rates and thus improved participation.

\section{Conflict of Interest statement}

No conflicts of interests.

\section{Acknowledgements}


We express our gratitude to all the corresponding or senior authors responding to our inquiries.

\section{Hosted file}

TABLE 1.pdf available at https://authorea.com/users/390710/articles/504948-factorsinfluencing-participation-rates-in-clinical-late-effect-studies-of-childhood-cancersurvivors

FIGURE 1. Flowchart of the selection of Nordic studies published from January $1^{\text {st }} 2010$ - March $1^{\text {st }} 2020$ according to inclusion and exclusion criteria

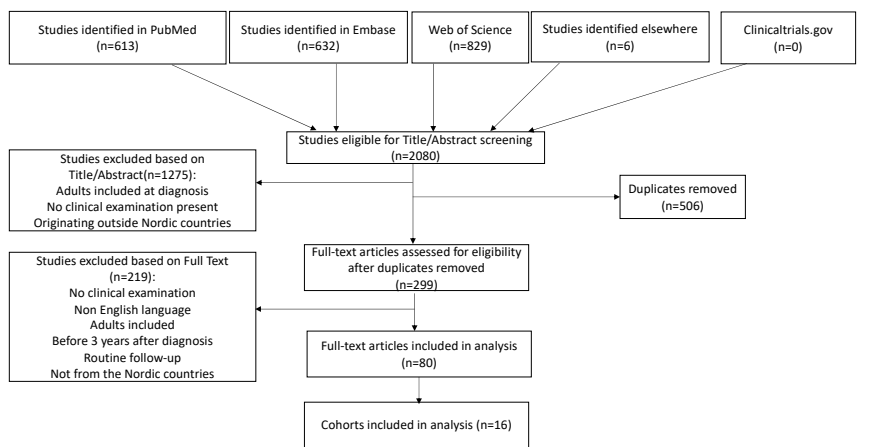




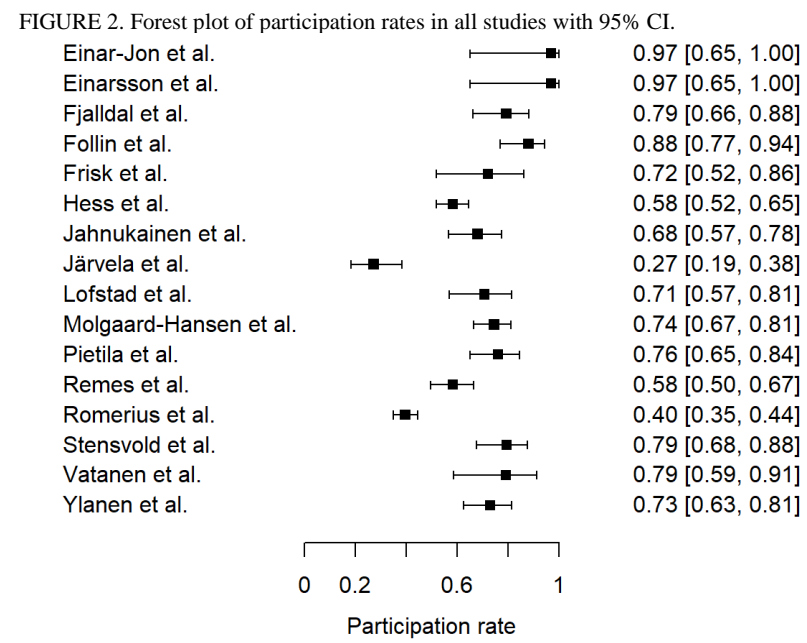




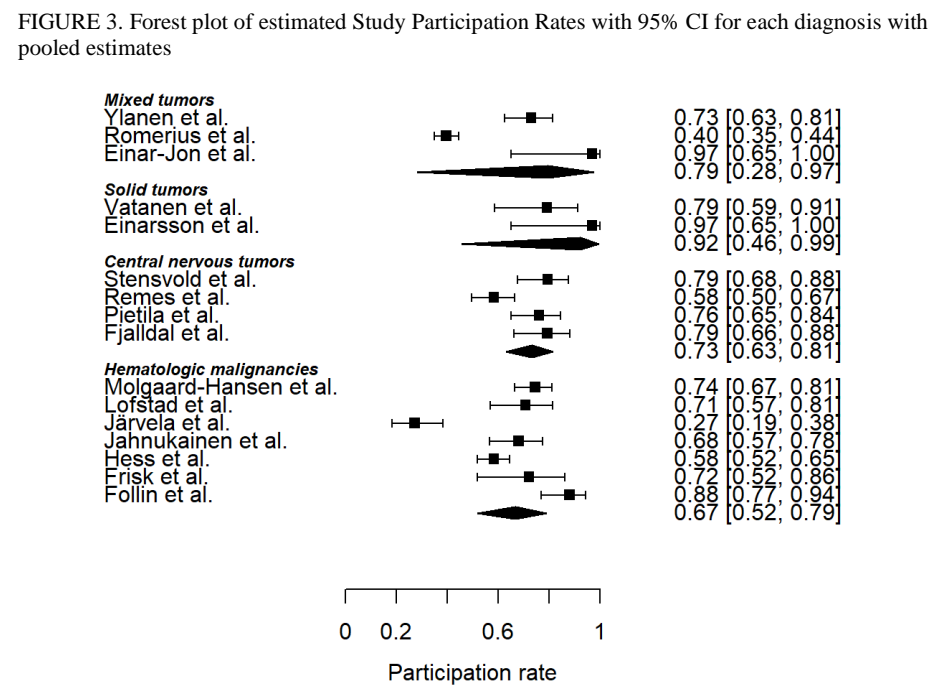




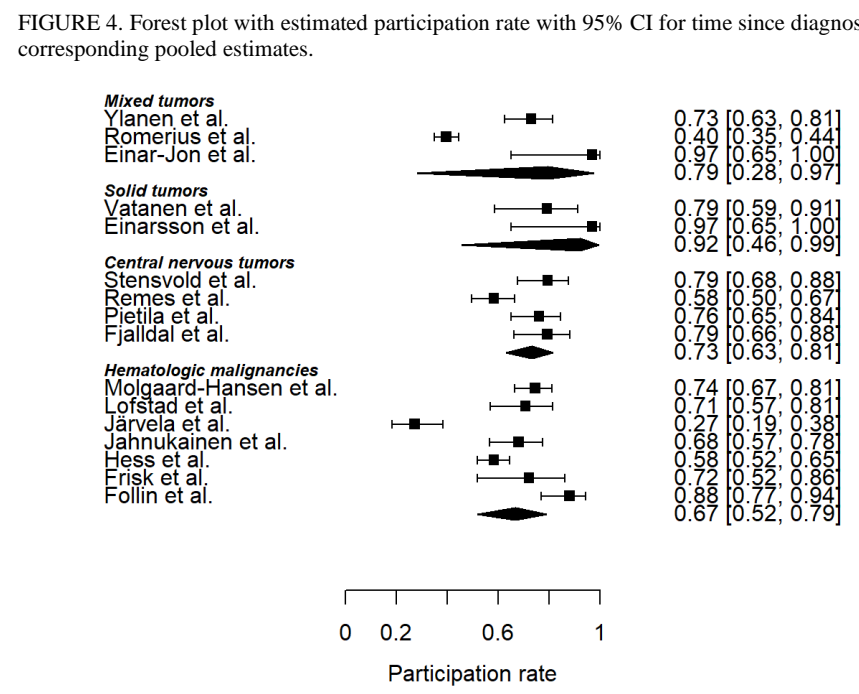




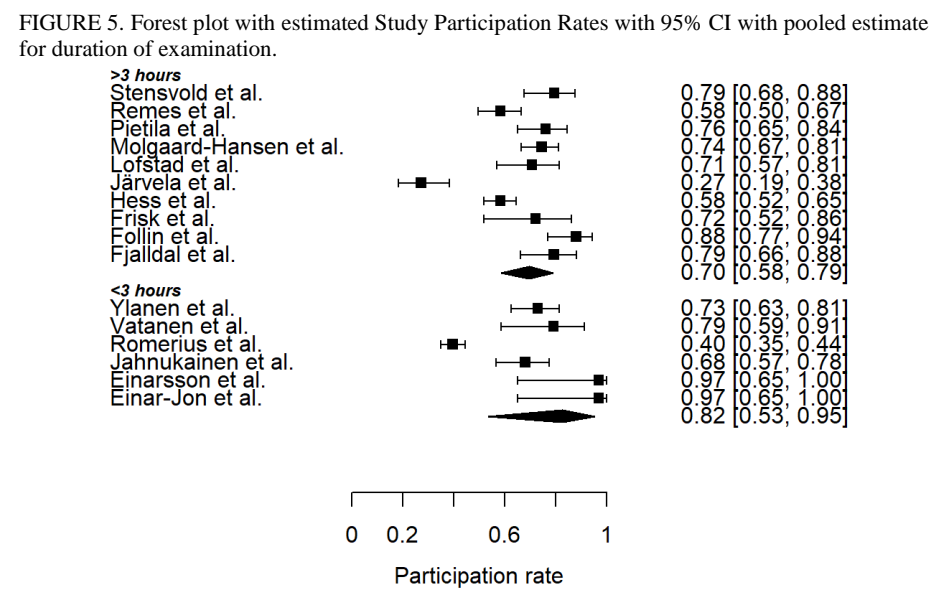

